\title{
Modular Hydrogen Peroxide Electrosynthesis Cell with Anthraquinone-Modified Polyaniline Electrocatalyst
}

\author{
Qianhong Zhu, Marlena Hinkle, David J. Kim, Jae-Hong Kim* \\ Department of Chemical and Environmental Engineering, Yale University, New Haven, CT \\ 06511, USA \\ *Corresponding Author. Email: jaehong.kim@yale.edu
}

\section{Section S1. Experimental Details}

\section{Text S1. Chemicals}

All chemicals were used as received without further purification. Aniline (emeraldine base) was purchased from Alfa Aesar; ammonium persulfate (ACS Reagent, $\geq 98 \%$ ), hydrogen chloride (ACS Reagent, 37\%), anthraquinone-2-carboxylic acid (98\%), dichloromethane (HPLC plus, $\geq 99.9 \%$ ), N-(3- dimethylaminopropyl)-N'-ethylcarbodiimide hydrochloride (EDC.HCl, crystalline), 1- hydroxybenzotriazole hydrate (HoBt hydrate, 97\%), N,N-diisopropylethylamine (DIEA, $\geq 99.9 \%$ ), isopropanol (ACS Reagent, $\geq 99.5 \%$ ), sodium phosphate monobasic monohydrate (BioXtra, $\geq 99.5 \%$ ), sodium phosphate dibasic (BioXtra, $\geq 99.0 \%$ ), ampliflu red, horseradish peroxide, $\mathrm{H}_{2} \mathrm{O}_{2}$ standard solution ( $30 \mathrm{wt} \%$ in $\mathrm{H}_{2} \mathrm{O}$, ACS reagent), methanol (for HPLC, $\geq 99.0 \%$ ), calcium sulfate dihydrate (ACS Reagent, 98\%), calcium chloride dihydrate (ACS Reagent, $\geq 99 \%$ ), sodium bicarbonate (ACS Reagent, $\geq 99.7 \%$ ), magnesium nitrate hexahydrate (ACS Reagent, $\geq 99 \%$ ), potassium phosphate monobasic (primary standard crystalline), magnesium sulfate, sodium silicate ( $\geq 95 \%$ ), sodium nitrate (ReagentPlus, $\geq 99 \%$ ), and sodium fluoride (ACS Reagent, $\geq 99 \%$ ) were obtained from Sigma Aldrich. $\mathrm{O}_{2}$ (99.996\%) were obtained from Airgas. All solutions were prepared with 18.2 M $\Omega$ Milli-Q deionized water.

\section{Text S2. AQ coverage calculation}

The chemical formula of green emeraldine salt is written as $\left(\mathrm{C}_{6} \mathrm{H}_{7} \mathrm{~N}\right)_{\mathrm{x}}$, and AQ-2-COOH is $\mathrm{C}_{15} \mathrm{H}_{8} \mathrm{O}_{4}$. After amide bond is formed, one hydrogen atom in PANI backbone and one -OH group in AQ-COOH are lost. As PANI contains $\mathrm{C}, \mathrm{N}, \mathrm{O}$, and $\mathrm{H}$, while AQ only contains $\mathrm{C}$, $\mathrm{O}$, and $\mathrm{H}$, the calculation will be based on the nitrogen balance. We also assume that AQ coverage on PANI surfaces is uniform over the XPS measurement area. Using PANI(RM) as an example, we can first assume AQ-PANI(RM) contains (1-x) mol of PANI and $x$ mol of AQ. According to XPS elemental analysis (Figure S3), the nitrogen balance can be written as $0.124 \times(1-\mathrm{x})=0.104$, so $\mathrm{x}=0.1613$. The mass coverage of AQ in AQ-PANI(RM) is $0.1613 \times(252-17) /((0.1613 \times(252-17)+(1$ $0.1613) \times 93-0.1613)=0.3275$. Therefore, the mol fraction of AQ is $16.13 \%$ and mass fraction is $32.75 \%$. Similar calculations can be applied to other samples and the results are summarized in Table 1. 


\section{Section S2. Additional graphs and tables}
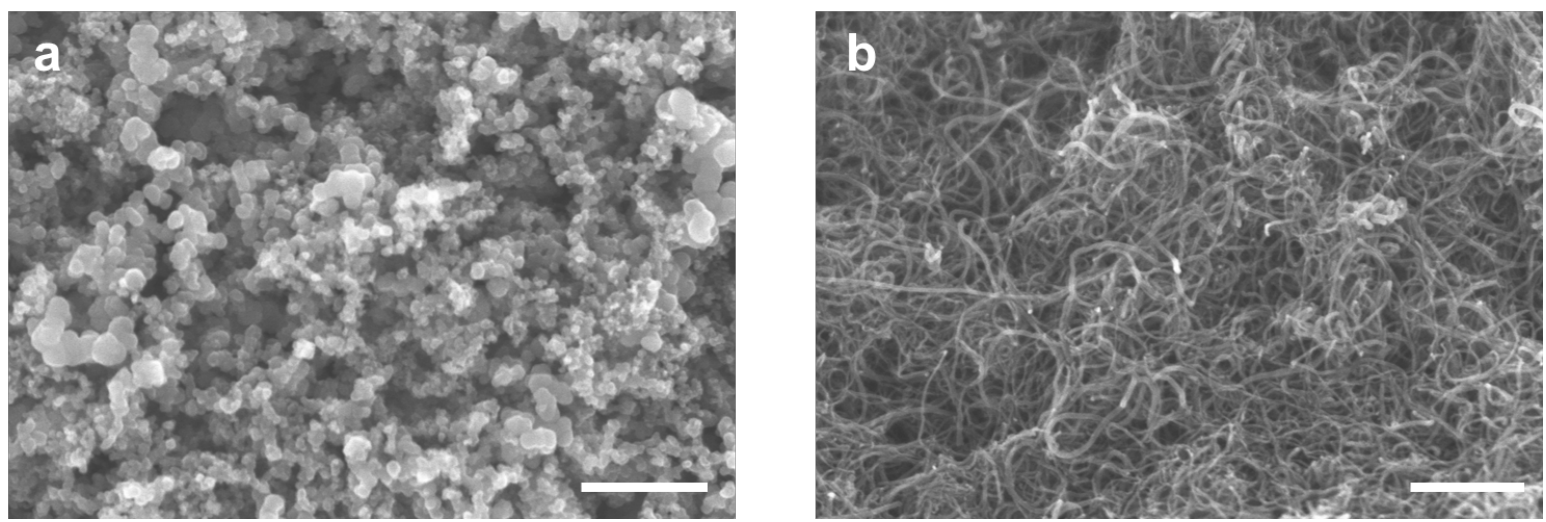

Figure S1. Additional SEM pictures of (a) carbon black (CB) and (b) carbon nanotube (CNT). The white bars at bottom right corner represent the scales of $500 \mathrm{~nm}$. CB had particle sizes of 50-120 nm; CNT had fiber lengths of 300$500 \mathrm{~nm}$ and widths of $10-20 \mathrm{~nm}$.

a

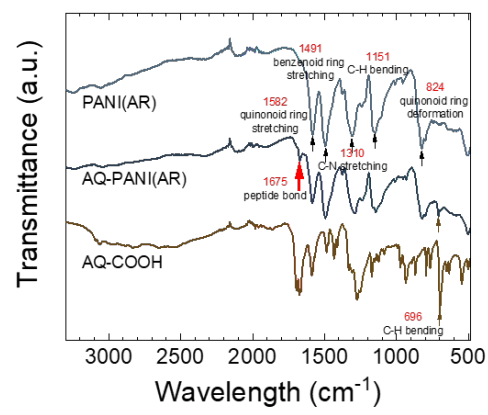

b

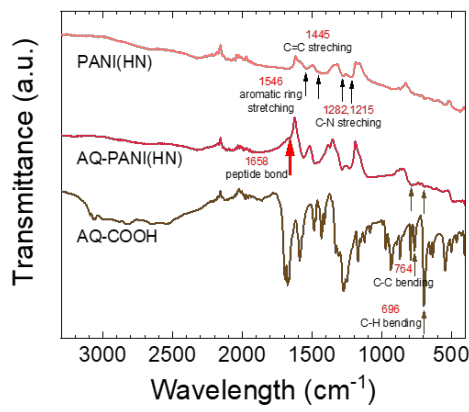

C

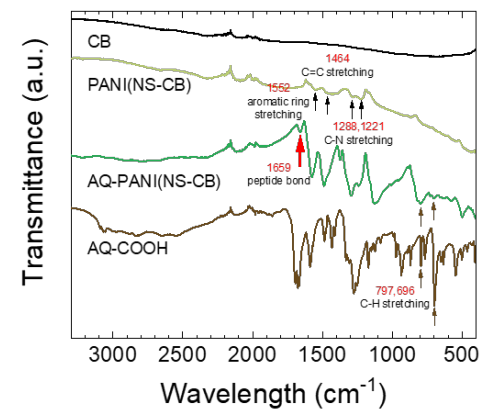

Figure S2. Additional FTIR spectra of (a) PANI(AR), AQ-PANI(AR), AQ-COOH, (b) PANI(HN), AQ-PANI(HN), $\mathrm{AQ}-\mathrm{COOH}$ and (c) carbon black (CB), PANI(NS-CB), AQ-PANI(NS-CB), and AQ-COOH.
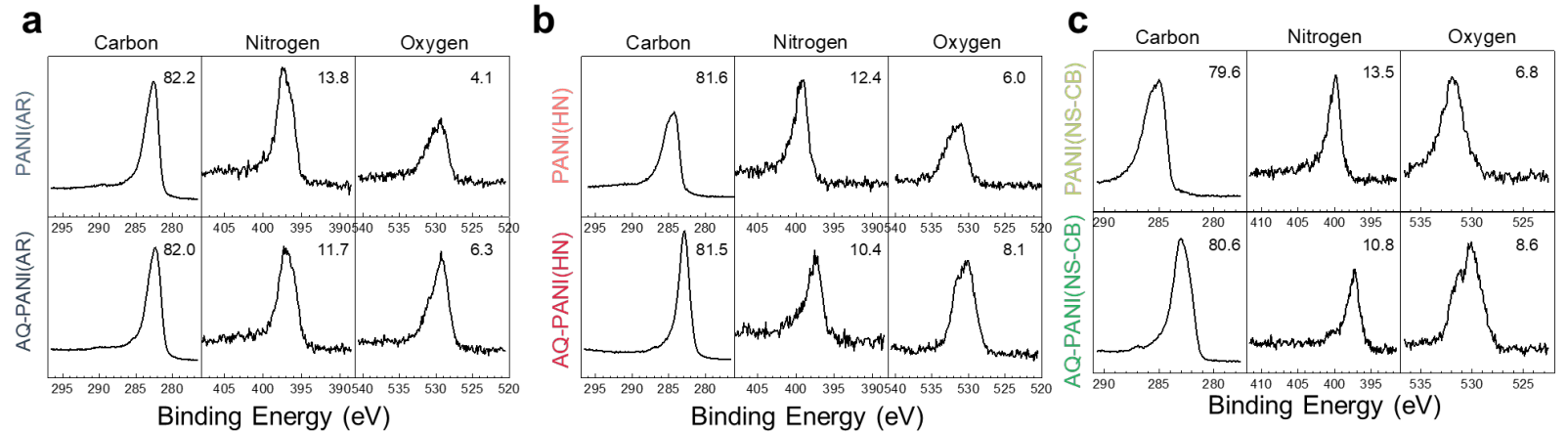

Figure S3. Additional XPS spectra of (a) PANI(AR), AQ-PANI(AR), (b) PANI(HN), AQ-PANI(HN), and (c) PANI(NS-CB), AQ-PANI(NS-CB) samples presenting their carbon 1s (first column), nitrogen 1s (second column), and oxygen 1s (third column) core levels. Numbers at the top right corner of each curve represent the atomic percentage of the corresponding element in each material, calculated by integrating peak area. Peak intensities are normalized to the strongest peak. 

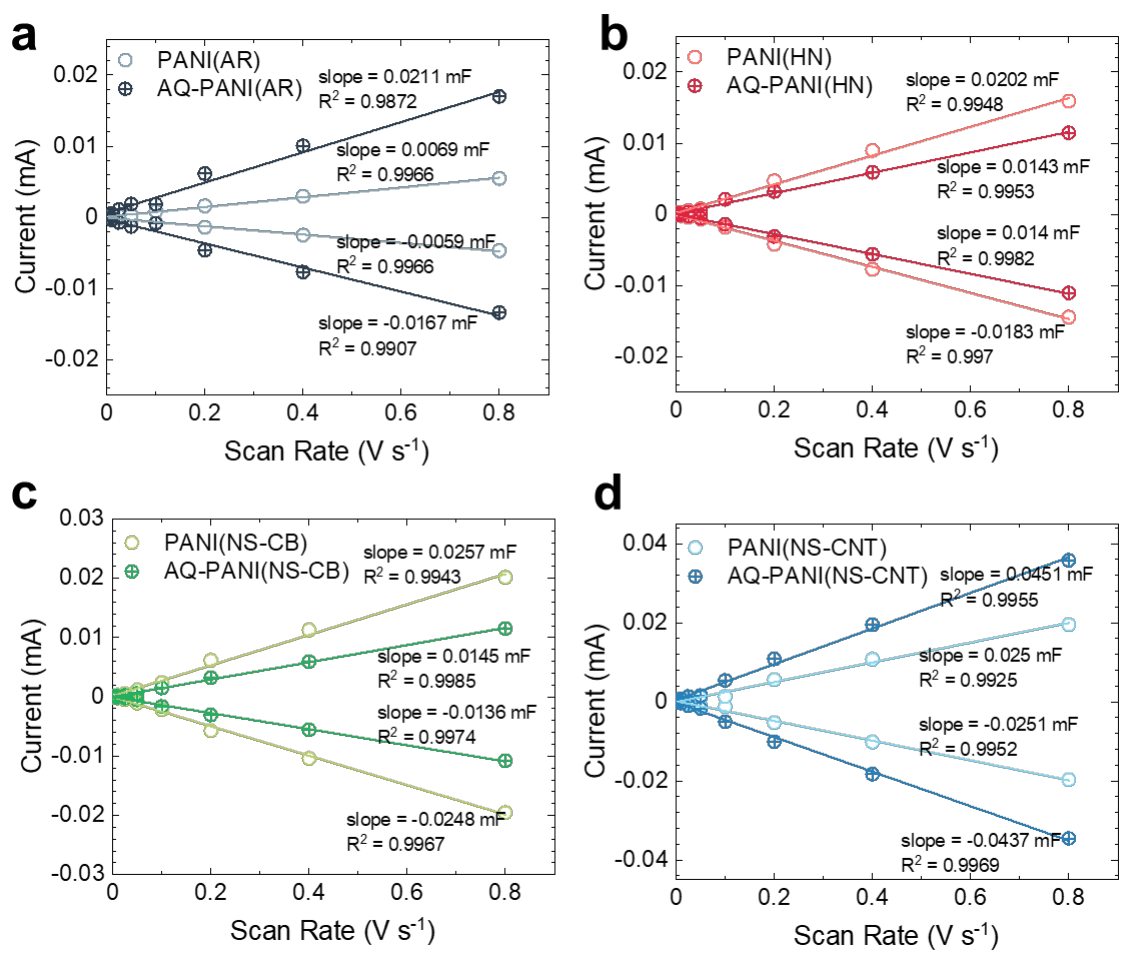

Figure S4. The cathodic and anodic charging currents measured at 0 to $0.2 \mathrm{~V}$ vs. $\mathrm{Ag} / \mathrm{AgCl}$ plotted as a function of scan rate for various PANI and AQ-PANI electrodes in $0.1 \mathrm{M}$ phosphate buffer of $\mathrm{pH} 8.8$.

\section{a}

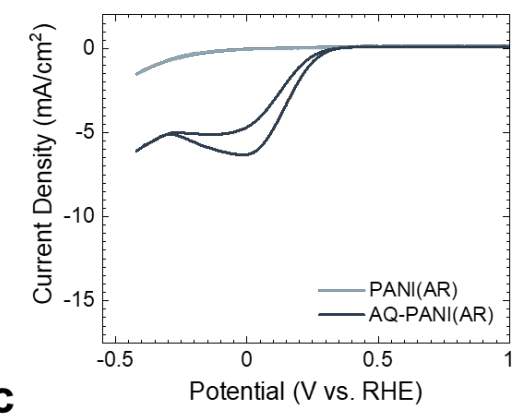

c

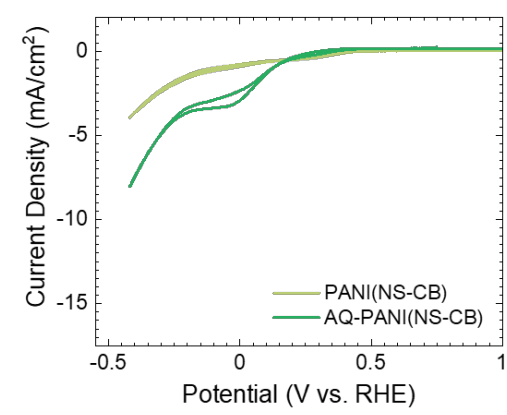

b

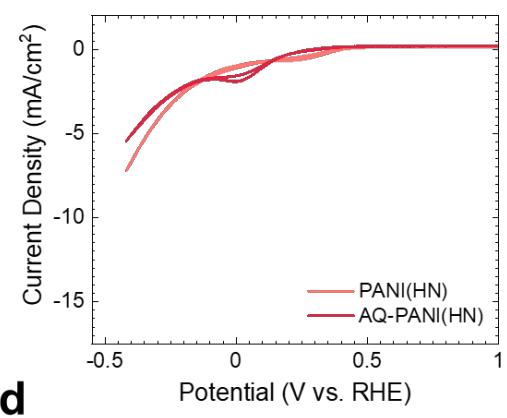

d

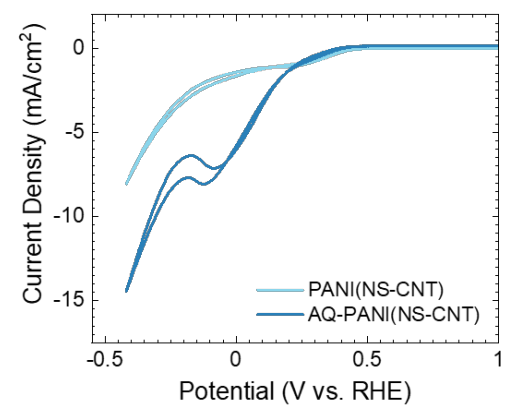

Figure S5. Cyclic voltammetry graphs of PANI and AQ-PANI electrodes at immersed electrode setup in $0.1 \mathrm{M} \mathrm{pH}=$ 9 phosphate buffer purged with $\mathrm{O}_{2}$. All the electrodes had the same area $\left(1 \mathrm{~cm}^{2}\right)$ and the same catalyst loading $(0.25$ $\mathrm{mg} \mathrm{cm}{ }^{-2}$ ). 
Table S1. Comparison of 2e- ORR electrocatalysts with anthraquinone or its derivatives

\begin{tabular}{|c|c|c|c|c|c|c|c|}
\hline \multirow[t]{2}{*}{ Substrate } & \multirow{2}{*}{$\begin{array}{l}\mathrm{H}_{2} \mathrm{O}_{2} \\
\text { generation } \\
\text { rate }\end{array}$} & \multirow{2}{*}{$\begin{array}{l}\text { Faradaic } \\
\text { efficiency } \\
(\%)\end{array}$} & \multicolumn{2}{|c|}{$\begin{array}{l}\mathrm{H}_{2} \mathrm{O}_{2} \text { generation } \\
\text { condition }\end{array}$} & \multirow{2}{*}{$\begin{array}{l}\text { Redox } \\
\text { potential } \\
\left(E_{1 / 2}, \text { Vvs. }\right. \\
\text { RHE) }\end{array}$} & \multirow[t]{2}{*}{$\begin{array}{l}\text { AQ coverage } \\
\text { density }\end{array}$} & \multirow[t]{2}{*}{ Binding strategy } \\
\hline & & & electrolyte & $\begin{array}{l}\text { Potential } \\
\text { (V. vs. } \\
\text { RHE) }\end{array}$ & & & \\
\hline $\begin{array}{l}\text { Glassy } \\
\text { carbon }^{1}\end{array}$ & N/A & $95-100$ & $0.1 \mathrm{M} \mathrm{KOH}$ & $\begin{array}{l}-0.7-- \\
1.25\end{array}$ & 0.17 & $\begin{array}{l}0.052-0.43 \\
\mathrm{nmol} \mathrm{cm}\end{array}$ & $\begin{array}{l}\text { Covalent attachment of } \mathrm{AQ} \text { by } \\
\text { reducing the } \mathrm{AQ} \text { diazonium salt }\end{array}$ \\
\hline $\begin{array}{l}\text { Indium tin } \\
\text { oxide }^{2}\end{array}$ & N/A & N/A & N/A & $\mathrm{N} / \mathrm{A}$ & 0.14 & $40-80 \mathrm{pmol} \mathrm{cm}^{-2}$ & $\begin{array}{l}\pi-\pi \text { interaction between pyrene- } \\
\text { bound ITO and pyrene-appended } \\
\text { anthraquinone }\end{array}$ \\
\hline $\begin{array}{l}\text { Graphene } \\
\text { oxide }^{3}\end{array}$ & N/A & $\sim 80$ & $0.1 \mathrm{M} \mathrm{KOH}$ & N/A & 0.17 & N/A & $\begin{array}{l}\text { Covalent attachment of AQ by } \\
\text { potentiodynamic electro- } \\
\text { reduction of Fast Red Al Salt }\end{array}$ \\
\hline $\begin{array}{l}\text { Carbon } \\
\text { nanotube }^{4}\end{array}$ & $\begin{array}{l}0.02 \mathrm{mmol}^{-2} \\
\mathrm{hr}^{-1} \mathrm{~cm}^{-2}\end{array}$ & $\sim 100$ & $1 \mathrm{M} \mathrm{KOH}$ & 1.0 & 0.10 & N/A & $\begin{array}{l}\text { Drop-cast AQ-2-COOH solution } \\
\text { onto carbon paper substrates }\end{array}$ \\
\hline $\begin{array}{l}\text { Gold disk } \\
\text { electrode }^{5}\end{array}$ & N/A & N/A & N/A & $\mathrm{N} / \mathrm{A}$ & 0.20 & $0.32 \mathrm{nmol} \mathrm{cm}^{-2}$ & $\begin{array}{l}\text { Self-assembled 1-hydroxy } \\
\text { anthraquinone disulfide } \\
\text { monolayer on gold disk } \\
\text { electrode via incubation }\end{array}$ \\
\hline $\begin{array}{l}\text { Carbon } \\
\text { black }^{6}\end{array}$ & $\begin{array}{l}0.52 \mathrm{mmol}^{-2} \\
\mathrm{hr}^{-1} \mathrm{~cm}^{-2}\end{array}$ & 89.6 & $\begin{array}{l}0.1 \mathrm{M} \mathrm{H}_{2} \mathrm{SO}_{4} \\
+0.1 \mathrm{M} \\
\mathrm{K}_{2} \mathrm{SO}_{4}\end{array}$ & -0.7 & 0.07 & $\begin{array}{l}1.0 \mathrm{wt} \% \text { (up to } \\
3.0 \mathrm{wt} \% \text { ) }\end{array}$ & $\begin{array}{l}\text { Tert-butyl-anthraquinone in } \\
\text { suspended solution with carbon } \\
\text { black deposited onto disk } \\
\text { electrode of RRDE }\end{array}$ \\
\hline $\begin{array}{l}\text { Polymeric } \\
\text { carbon } \\
\text { nitride }^{7}\end{array}$ & $\begin{array}{l}60.08 \mathrm{mmol}^{-1} \\
\mathrm{~g}_{\text {catalyst }}{ }^{-h^{-1}}\end{array}$ & 42.2 & $\begin{array}{l}0.5 \mathrm{M} \\
\text { phosphate } \\
\text { buffer (pH 9) }\end{array}$ & -0.2 & 0.27 & $\begin{array}{l}20 \mathrm{wt}^{2}, 377 \\
\mathrm{nmol} \mathrm{cc^{-2 }}(0.5 \\
\mathrm{mg} \mathrm{cm} \text { catalyst })^{-2}\end{array}$ & $\begin{array}{l}\text { Chemical bond between } \mathrm{C}_{3} \mathrm{~N}_{4}(- \\
\left.\mathrm{NH}_{2} /-\mathrm{NH}\right) \text { and } \mathrm{AQ}-\mathrm{COOH}(- \\
\mathrm{COOH})\end{array}$ \\
\hline $\begin{array}{l}\text { Poly- } \\
\text { aniline } \\
\text { (this } \\
\text { work) }\end{array}$ & $\begin{array}{l}1.8 \mathrm{~mol} \\
\mathrm{~g}_{\text {catalyst }}{ }^{-1} \mathrm{hr}^{-1}\end{array}$ & 95.8 & $\begin{array}{l}0.1 \mathrm{M} \\
\text { phosphate } \\
\text { buffer }(\mathrm{pH} \\
8.8)\end{array}$ & -0.02 & 0.05 & $\begin{array}{l}32.33 \mathrm{wt}^{\circ} \%, 320.5 \\
\mathrm{nmol} \mathrm{\textrm {cm } ^ { - 2 }}(0.25 \\
\left.\mathrm{mg} \mathrm{cm}^{-2} \text { catalyst }\right)\end{array}$ & $\begin{array}{l}\text { Chemical bond between } \\
\text { polyaniline (-NH) and AQ- } \\
\mathrm{COOH}(-\mathrm{COOH})\end{array}$ \\
\hline
\end{tabular}

Table S2. Energy consumptions of $90 \%$ micropollutant degradation via $\mathrm{H}_{2} \mathrm{O}_{2} / \mathrm{UV}$ process

\begin{tabular}{|c|c|c|c|c|c|c|c|c|}
\hline Target compound & $\begin{array}{l}\text { Target } \\
\text { compound } \\
\text { concentrati } \\
\text { on }\end{array}$ & Water matrix & $\mathrm{pH}$ & $\begin{array}{l}\text { Energy } \\
\text { consump- } \\
\text { tion for UV } \\
\left(\mathrm{E}_{\mathrm{UV}}, \mathrm{kwh}\right. \\
\left.\mathrm{m}^{-3}\right)\end{array}$ & $\begin{array}{l}\mathrm{H}_{2} \mathrm{O}_{2} \\
\text { concentra- } \\
\text { tion }\end{array}$ & $\begin{array}{l}\text { Energy } \\
\text { consumpti } \\
\text { on for } \\
\mathrm{H}_{2} \mathrm{O}_{2} \\
\text { generation } \\
\left(\mathrm{E}_{\mathrm{H} 2 \mathrm{O} 2}\right. \\
\text { kwh m } \\
\text {-3 }) \\
\end{array}$ & $\begin{array}{l}\mathrm{E}_{\mathrm{H} 2 \mathrm{O} 2} /(\mathrm{E} \\
\mathrm{H}_{2} \mathrm{O} 2+\mathrm{E}_{\mathrm{UV}} \\
)(\%)^{*}\end{array}$ & Ref \\
\hline Sulfamethoxazole & \multirow[t]{4}{*}{$0.5 \mu \mathrm{M}$} & \multirow{4}{*}{$\begin{array}{l}\text { Natural } \\
\text { surface water } \\
\text { (Lake Zurich) }\end{array}$} & \multirow[t]{4}{*}{8} & 0.39 & \multirow[t]{4}{*}{$0.2 \mathrm{mM}$} & \multirow[t]{4}{*}{0.04} & 9.09 & \multirow[t]{4}{*}{8} \\
\hline$p$-chlorobenzoic acid & & & & 0.75 & & & 5.06 & \\
\hline Atrazine & & & & 0.98 & & & 3.92 & \\
\hline $\begin{array}{l}\mathrm{N}- \\
\text { Nitrosodimethylamine }\end{array}$ & & & & 1.62 & & & 2.41 & \\
\hline Methylparaben & \multirow[t]{11}{*}{$1 \mu \mathrm{g} \mathrm{L}^{-1}$} & \multirow{11}{*}{$\begin{array}{l}\text { Wastewater } \\
\text { treatment } \\
\text { plant effluent }\end{array}$} & \multirow[t]{11}{*}{3} & 8.1 & \multirow[t]{11}{*}{$60 \mathrm{mg} \mathrm{L}^{-1}$} & \multirow[t]{11}{*}{0.34} & 4.03 & \multirow[t]{11}{*}{9} \\
\hline Ethylparaben & & & & 5.3 & & & 6.03 & \\
\hline Propylparaben & & & & 6.4 & & & 5.04 & \\
\hline isoButylparaben & & & & 7.0 & & & 4.63 & \\
\hline Butylparaben & & & & 7.9 & & & 4.13 & \\
\hline Bisphenol A & & & & 8.7 & & & 3.76 & \\
\hline isoNonylphenol & & & & 7.6 & & & 4.28 & \\
\hline Octylphenol & & & & 2.6 & & & 11.56 & \\
\hline Benzophenone-3 & & & & 8.5 & & & 3.85 & \\
\hline Benzophenone-7 & & & & 8.7 & & & 3.76 & \\
\hline $\begin{array}{l}\text { Octyl } \\
\text { methoxycinnamate }\end{array}$ & & & & 7.1 & & & 4.57 & \\
\hline
\end{tabular}




\begin{tabular}{|c|c|c|c|c|c|c|c|c|}
\hline Homosalate & & & & 7.7 & & & 4.23 & \\
\hline $\begin{array}{l}\text { 3-(4-methylbenzyliden) } \\
\text { camphor }\end{array}$ & & & & 5.0 & & & 6.37 & \\
\hline $\begin{array}{l}\text { Octyl } \\
\text { dimethylaminobenzoate }\end{array}$ & \multirow{3}{*}{$400 \mathrm{ng} \mathrm{L}^{-1}$} & & & 4.1 & & & 7.66 & \\
\hline $17 \beta$-estradiol & & & & 2.2 & & & 13.39 & \\
\hline ethinylestradiol & & & & 1.8 & & & 15.89 & \\
\hline Caffeine & \multirow[t]{13}{*}{$50 \mu \mathrm{g} \mathrm{L}^{-1}$} & \multirow{13}{*}{$\begin{array}{l}\text { Pre-treated } \\
\text { wastewater } \\
\text { treatment } \\
\text { plant effluent }\end{array}$} & \multirow[t]{13}{*}{7.1} & 7.0 & \multirow[t]{7}{*}{$10 \mathrm{mg} \mathrm{L}^{-1}$} & \multirow[t]{7}{*}{0.06} & 0.81 & \multirow[t]{13}{*}{10} \\
\hline Carbamazepine & & & & 3.9 & & & 1.45 & \\
\hline Naproxen & & & & 1.3 & & & 4.23 & \\
\hline Clofibric acid & & & & 0.7 & & & 7.59 & \\
\hline $17 \beta$-estradiol & & & & 1.3 & & & 4.23 & \\
\hline Sulphamethoxazole & & & & 0.8 & & & 6.70 & \\
\hline Diclofenac & & & & 0.3 & & & 16.08 & \\
\hline Caffeine & & & & 4.4 & \multirow[t]{6}{*}{$20 \mathrm{mg} \mathrm{L}^{-1}$} & \multirow[t]{6}{*}{0.12} & 2.55 & \\
\hline Carbamazepine & & & & 2.5 & & & 4.40 & \\
\hline Naproxen & & & & 1.2 & & & 8.75 & \\
\hline Clofibric acid & & & & 0.8 & & & 12.57 & \\
\hline $17 \beta$-estradiol & & & & 1.3 & & & 8.13 & \\
\hline Sulphamethoxazole & & & & 0.8 & & & 12.57 & \\
\hline Dimetridazole & \multirow[t]{28}{*}{$1 \mu \mathrm{g} \mathrm{L}^{-1}$} & \multirow{28}{*}{$\begin{array}{l}\text { Simulated } \\
\text { drinking } \\
\text { water }\end{array}$} & \multirow[t]{28}{*}{7} & 1.74 & \multirow[t]{28}{*}{$20 \mu \mathrm{M}$} & \multirow[t]{28}{*}{0.004} & 0.22 & \multirow[t]{39}{*}{11} \\
\hline Tinidazole & & & & 2.46 & & & 0.16 & \\
\hline Omidazole & & & & 2.01 & & & 0.19 & \\
\hline Metronidazole & & & & 1.93 & & & 0.20 & \\
\hline Ronidazole & & & & 2.34 & & & 0.17 & \\
\hline Ibuprofen & & & & 1.51 & & & 0.26 & \\
\hline Chloramphenicol & & & & 1.45 & & & 0.27 & \\
\hline Primidone & & & & 1.35 & & & 0.29 & \\
\hline Venlafaxine & & & & 1.13 & & & 0.34 & \\
\hline Nalidixic acid & & & & 1.18 & & & 0.33 & \\
\hline Flumequine & & & & 1.42 & & & 0.27 & \\
\hline Atenolol & & & & 1.39 & & & 0.28 & \\
\hline Metoprolol & & & & 1.28 & & & 0.30 & \\
\hline Erythromycin & & & & 1.80 & & & 0.22 & \\
\hline Azithromycin & & & & 1.93 & & & 0.20 & \\
\hline Roxithromycin & & & & 2.09 & & & 0.19 & \\
\hline Carbamazepine & & & & 1.16 & & & 0.34 & \\
\hline Caffeine & & & & 1.75 & & & 0.22 & \\
\hline Gemfibrozil & & & & 1.41 & & & 0.28 & \\
\hline Naproxen & & & & 1.11 & & & 0.35 & \\
\hline Propranolol & & & & 0.76 & & & 0.51 & \\
\hline Clenbuterol & & & & 1.66 & & & 0.23 & \\
\hline Diclofenac & & & & 0.17 & & & 2.25 & \\
\hline Trimethoprim & & & & 1.18 & & & 0.33 & \\
\hline Salbutamol & & & & 1.85 & & & 0.21 & \\
\hline Ractopamine & & & & 0.96 & & & 0.41 & \\
\hline Bisphenol A & & & & 1.06 & & & 0.37 & \\
\hline Sulfamethoxazole & & & & 0.42 & & & 0.92 & \\
\hline Dimetridazole & \multirow[t]{11}{*}{$1 \mu \mathrm{g} \mathrm{L}^{-1}$} & & 7 & 3.36 & $50 \mu \mathrm{M}$ & 0.01 & 0.29 & \\
\hline Tinidazole & & wastewater & & 3.36 & & & 0.29 & \\
\hline Omidazole & & treatment & & 4.50 & & & 0.22 & \\
\hline Metronidazole & & plant effluent & & 6.79 & & & 0.14 & \\
\hline Ronidazole & & & & 3.36 & & & 0.29 & \\
\hline Ibuprofen & & & & 6.71 & & & 0.15 & \\
\hline Chloramphenicol & & & & 1.54 & & & 0.63 & \\
\hline Primidone & & & & 2.18 & & & 0.45 & \\
\hline Venlafaxine & & & & 2.71 & & & 0.36 & \\
\hline Nalidixic acid & & & & 0.71 & & & 1.36 & \\
\hline Flumequine & & & & 2.54 & & & 0.38 & \\
\hline
\end{tabular}




\begin{tabular}{|c|c|c|c|c|c|c|c|c|}
\hline Atenolol & & & & 2.54 & & & 0.38 & \\
\hline Metoprolol & & & & 2.21 & & & 0.44 & \\
\hline Erythromycin & & & & 5.82 & & & 0.17 & \\
\hline Azithromycin & & & & 8.07 & & & 0.12 & \\
\hline Roxithromycin & & & & 3.43 & & & 0.28 & \\
\hline Carbamazepine & & & & 3.39 & & & 0.29 & \\
\hline Caffeine & & & & 1.93 & & & 0.50 & \\
\hline Gemfibrozil & & & & 8.14 & & & 0.12 & \\
\hline Naproxen & & & & 2.54 & & & 0.38 & \\
\hline Propranolol & & & & 1.71 & & & 0.57 & \\
\hline Clenbuterol & & & & 1.32 & & & 0.73 & \\
\hline Diclofenac & & & & 0.25 & & & 3.76 & \\
\hline Trimethoprim & & & & 1.43 & & & 0.68 & \\
\hline Salbutamol & & & & 2.61 & & & 0.37 & \\
\hline Ractopamine & & & & 1.54 & & & 0.63 & \\
\hline Bisphenol A & & & & 1.47 & & & 0.66 & \\
\hline Sulfamethoxazole & & & & 0.61 & & & 1.58 & \\
\hline Enrofloxacin & \multirow[t]{2}{*}{$1 \mathrm{~g} \mathrm{~L}^{-1}$} & \multirow[t]{3}{*}{ Surface water } & \multirow[t]{3}{*}{7.0} & 91.7 & \multirow[t]{2}{*}{$20 \mathrm{mM}$} & \multirow[t]{2}{*}{3.91} & 4.09 & \multirow[t]{3}{*}{12} \\
\hline Pefloxacin & & & & 176.1 & & & 2.17 & \\
\hline Sulfaquinoxaline & $4 \mathrm{mg} \mathrm{L}^{-1}$ & & & 63.7 & $5 \mathrm{mM}$ & 0.98 & 1.52 & \\
\hline \multirow[t]{6}{*}{ Sulfolane } & \multirow[t]{4}{*}{$200 \mu \mathrm{g} \mathrm{L}^{-1}$} & \multirow[t]{6}{*}{ Groundwater } & \multirow[t]{4}{*}{8.5} & 4.0 & \multirow[t]{2}{*}{$40 \mathrm{mg} \mathrm{L}^{-1}$} & \multirow[t]{2}{*}{0.23} & 5.44 & \multirow[t]{6}{*}{13} \\
\hline & & & & 3.6 & & & 6.01 & \\
\hline & & & & 2.6 & \multirow[t]{2}{*}{$80 \mathrm{mg} \mathrm{L}^{-1}$} & \multirow[t]{2}{*}{0.46} & 15.03 & \\
\hline & & & & 2.5 & & & 15.54 & \\
\hline & $260 \mu \mathrm{g} \mathrm{L}^{-1}$ & & 7.8 & 7.5 & \multirow[t]{2}{*}{$30 \mathrm{mg} \mathrm{L}^{-1}$} & \multirow[t]{2}{*}{0.17} & 2.22 & \\
\hline & $12.6 \mathrm{mg} \mathrm{L}^{-1}$ & & 9.1 & 4.0 & & & 4.08 & \\
\hline Atrazine & \multirow[t]{3}{*}{$10 \mu \mathrm{g} \mathrm{L}^{-1}$} & Surface water & 8.0 & 0.54 & $10 \mathrm{ppm}$ & 0.06 & 9.62 & 14 \\
\hline Bromacil & & & & 0.52 & & & 9.95 & \\
\hline Ibuprofen & & & & 0.50 & & & 10.31 & \\
\hline $\begin{array}{l}\mathrm{N}- \\
\text { nitrosodimethylamine }\end{array}$ & $20 \mu \mathrm{g} \mathrm{L}^{-1}$ & & & 0.30 & & & 16.08 & \\
\hline Atrazine & $10 \mu \mathrm{g} \mathrm{L}^{-1}$ & & & 0.73 & $5 \mathrm{ppm}$ & 0.03 & 3.79 & \\
\hline Bromacil & & & & 0.91 & & & 3.06 & \\
\hline Ibuprofen & & & & 0.74 & & & 3.74 & \\
\hline $\begin{array}{l}\mathrm{N}- \\
\text { nitrosodimethylamine }\end{array}$ & $20 \mu \mathrm{g} \mathrm{L}^{-1}$ & & & 0.30 & & & 8.74 & \\
\hline 1,1,1-trichloorethane & $5-10 \mu g$ & & & 0.23 & & & 11.10 & \\
\hline $\begin{array}{l}\text { 2,4-chloorfenoxy acetic } \\
\text { acid }\end{array}$ & $\mathrm{L}^{-1}$ & & & 1.45 & & & 1.94 & \\
\hline Bentazone & & & & 1.51 & & & 1.87 & \\
\hline Carbendazim & & & & 0.63 & & & 4.36 & \\
\hline Clofibrinic acid & & & & 0.54 & & & 5.05 & \\
\hline Diclofenac & & & & 0.15 & & & 16.08 & \\
\hline isoproturon & & & & 1.09 & & & 2.57 & \\
\hline $\begin{array}{l}\text { 2-methyl-4-chloro- } \\
\text { phenoxy acetic acid }\end{array}$ & & & & 0.53 & & & 5.14 & \\
\hline $\begin{array}{l}\text { Methyl-tertiary- } \\
\text { butylether }\end{array}$ & & & & 0.64 & & & 4.30 & \\
\hline Metolachlor & & & & 0.38 & & & 7.03 & \\
\hline $\begin{array}{l}\text { Tertiary-amyl- } \\
\text { methylether }\end{array}$ & & & & 0.26 & & & 9.95 & \\
\hline Methyl tert-butyl ether & $2.34 \mathrm{mg} \mathrm{L}^{-1}$ & Groundwater & 7.0 & 1.4 & $25 \mathrm{mg} \mathrm{L}^{-1}$ & 0.14 & 9.31 & 15 \\
\hline & & & 9.0 & 4.8 & & & 2.91 & \\
\hline & & & 7.0 & 1.2 & $50 \mathrm{mg} \mathrm{L}^{-1}$ & 0.29 & 19.32 & \\
\hline & & & 9.0 & 3.8 & & & 7.03 & \\
\hline & $0.963 \mathrm{mg}$ & & 7.0 & 3.9 & $25 \mathrm{mg} \mathrm{L}^{-1}$ & 0.14 & 3.55 & \\
\hline & $\mathrm{L}^{-1}$ & & 9.0 & 8.2 & & & 1.72 & \\
\hline & & & 7.0 & 3.9 & $50 \mathrm{mg} \mathrm{L}^{-1}$ & 0.29 & 6.86 & \\
\hline & & & 9.0 & 5.5 & & & 4.96 & \\
\hline
\end{tabular}




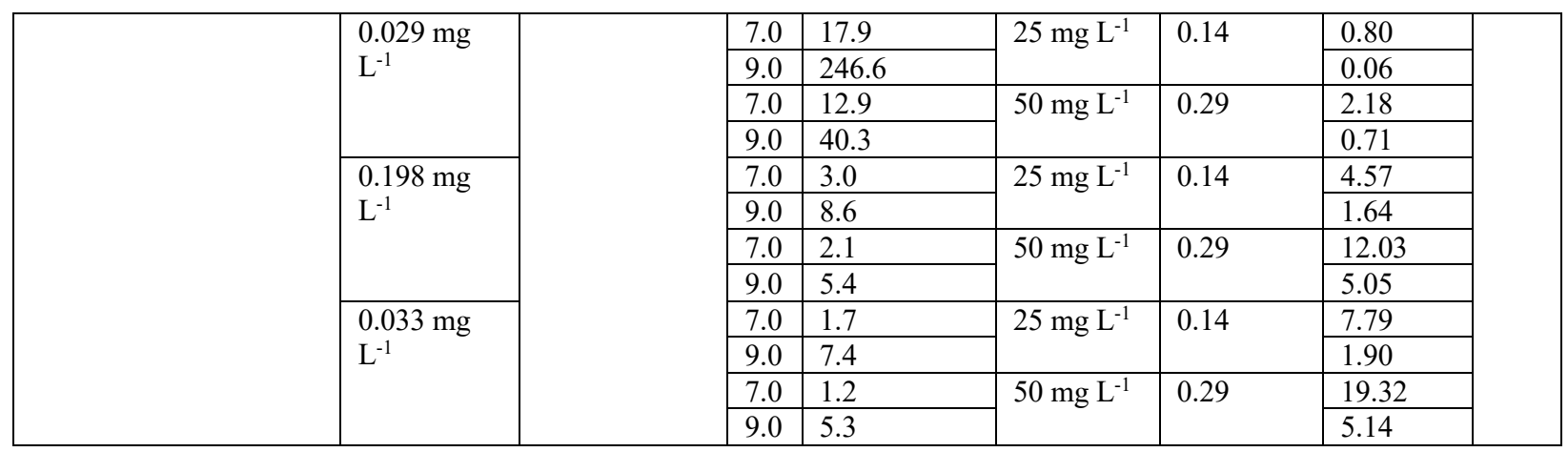

*. Energy was calculated based on cell conditions in simulated drinking water: cell current $=4 \mathrm{~mA}$, cell potential $=$ 3.6 V. At the same current density, it required the highest cell potential to drive the reaction in simulated drinking water, suggesting the highest energy requirement per unit mass of produced $\mathrm{H}_{2} \mathrm{O}_{2}$ in this condition. The energy consumption, as well as the ratio in overall process, should be lower in other water matrices than those in this table.

Table S3. Compositions of the water matrices

\begin{tabular}{|c|c|c|c|}
\hline & Synthetic drinking water & Simulated wastewater* & Electrolyte \\
\hline $\mathrm{pH}$ & 7.5 & 7.0 & 8.8 \\
\hline Conductivity $\left(\mathrm{mS} \mathrm{cm}^{-1}\right)$ & 0.865 & 5.48 & 12.57 \\
\hline \multicolumn{4}{|l|}{ Ions $\left(\mathrm{mg} \mathrm{L}^{-1}\right)$} \\
\hline Bicarbonate $\left(\mathrm{HCO}_{3}^{-}\right)$ & 183 & 0 & 0 \\
\hline Calcium $\left(\mathrm{Ca}^{2+}\right)$ & 40 & 0 & 0 \\
\hline Chloride $\left(\mathrm{Cl}^{-}\right)$ & 71 & 0 & 0 \\
\hline $\begin{array}{l}\text { Dihydrogen phosphate } \\
\left(\mathrm{H}_{2} \mathrm{PO}_{4}^{-}\right)\end{array}$ & 0 & 0 & 160 \\
\hline Fluoride $\left(\mathrm{F}^{-}\right)$ & 1.0 & 0 & \\
\hline $\begin{array}{l}\text { Hydrogenphosphate } \\
\left(\mathrm{HPO}_{4}^{2-}\right)\end{array}$ & 0 & 0 & 9330 \\
\hline Magnesium $\left(\mathrm{Mg}^{2+}\right)$ & 12 & 0 & 0 \\
\hline Nitrate $\left(\mathrm{NO}_{3}^{-}\right)$ & 8.9 & 0 & 0 \\
\hline Phosphate $\left(\mathrm{PO}_{4}^{-}\right)$ & 0.12 & 0 & 0 \\
\hline Silica $\left(\mathrm{SiO}_{2}\right)$ & 20 & 0 & 0 \\
\hline Sodium $\left(\mathrm{Na}^{+}\right)$ & 89 & 1620 & 4510 \\
\hline Sulfate $\left(\mathrm{SO}_{4}{ }^{2-}\right)$ & 48 & 3380 & 0 \\
\hline Total Diss. Solids (TDS) & 478 & 5000 & 14000 \\
\hline
\end{tabular}

*. Due to the complexity of wastewater compositions from various sources, a simple recipe of $5000 \mathrm{mg} \mathrm{L}^{-1} \mathrm{Na}_{2} \mathrm{SO}_{4}$ solution was used to simulate the ionic strength of wastewater. ${ }^{16-18}$

Table S4. Comparison of 2e- ORR electrocatalysts's stabilities

\begin{tabular}{|c|c|c|c|c|c|c|}
\hline \multirow{2}{*}{$\begin{array}{c}\text { Catalyst / } \\
\text { Electrode }\end{array}$} & \multicolumn{3}{|c|}{ Testing conditions } & \multicolumn{2}{c|}{ Performance metrics } & \multirow{2}{*}{ Ref. } \\
\cline { 2 - 6 } & Electrolyte & Operation & Duration (h) & Before testing & After testing & \\
\hline $\begin{array}{c}\text { Graphite }+ \\
\text { carbon black }\end{array}$ & $\begin{array}{c}0.05 \mathrm{M} \mathrm{Na}_{2} \mathrm{SO}_{4} \\
+100 \mathrm{mg} \mathrm{L}^{-1} \\
\text { phenol } \\
(\mathrm{pH} \mathrm{3})\end{array}$ & $10 \mathrm{~mA} \mathrm{~cm}^{-2}$ & 200 & {$\left[\mathrm{H}_{2} \mathrm{O}_{2}\right]: 316 \mathrm{mg} \mathrm{L}^{-1}$} & $\begin{array}{c}260 \mathrm{mg} \mathrm{L}^{-1} \\
\left(17.8 \% \mathrm{loss}^{2}\right.\end{array}$ & 19 \\
\hline
\end{tabular}




\begin{tabular}{|c|c|c|c|c|c|c|}
\hline Carbon black & $\begin{array}{c}\text { Tap water }+ \\
0.005 \mathrm{M} \\
\mathrm{Na}_{2} \mathrm{SO}_{4}\end{array}$ & $1.5 \mathrm{~mA} \mathrm{~cm}^{-2}$ & 1200 & {$\left[\mathrm{H}_{2} \mathrm{O}_{2}\right]: 9.8 \mathrm{mg} \mathrm{L}^{-1}$} & $9.8 \mathrm{mg} \mathrm{L}^{-1}$ & 20 \\
\hline $\begin{array}{l}\mathrm{CoS}_{\mathrm{x}} \mathrm{P}_{\mathrm{y}} \text { on } \\
\text { MWCNTs }\end{array}$ & $\begin{array}{c}0.05 \mathrm{M} \mathrm{Na}_{2} \mathrm{SO}_{4} \\
(\mathrm{pH} \mathrm{3})\end{array}$ & $25 \mathrm{~mA} \mathrm{~cm}^{-2}$ & 6 & $\begin{array}{c}\text { Current efficiency: } \\
85 \%\end{array}$ & $51-54 \%$ & 21 \\
\hline $\begin{array}{l}\text { WO on carbon } \\
\text { black }\end{array}$ & $0.1 \mathrm{M} \mathrm{K}_{2} \mathrm{SO}_{4}$ & $\begin{array}{c}-0.7 \mathrm{~V} \\
\text { vs. } \mathrm{Ag} / \mathrm{AgCl}\end{array}$ & 37 & {$\left[\mathrm{H}_{2} \mathrm{O}_{2}\right]: 479 \mathrm{mg} \mathrm{L}^{-1}$} & $\begin{array}{l}448 \mathrm{mg} \mathrm{L}^{-1} \\
(6.5 \% \text { loss })\end{array}$ & 22 \\
\hline $\begin{array}{l}\text { Oxidized } \\
\text { graphite felt }\end{array}$ & $\begin{array}{c}0.05 \mathrm{M} \mathrm{Na}_{2} \mathrm{SO}_{4} \\
(\mathrm{pH} \mathrm{3})\end{array}$ & $16.7 \mathrm{~mA} \mathrm{~cm}^{-2}$ & $\begin{array}{c}15 \\
(10 \text { runs of } 1.5 \\
h)\end{array}$ & {$\left[\mathrm{H}_{2} \mathrm{O}_{2}\right]: 34.4 \mathrm{mg} \mathrm{L}^{-1}$} & $\begin{array}{c}16.3 \mathrm{mg} \mathrm{L}^{-1} \\
(47.47 \% \text { loss })\end{array}$ & 23 \\
\hline $\begin{array}{c}\text { Activated } \\
\text { carbon }\end{array}$ & $\begin{array}{c}0.05 \mathrm{M} \mathrm{Na}_{2} \mathrm{SO}_{4} \\
(\mathrm{pH} \mathrm{7})\end{array}$ & $12.5 \mathrm{~mA} \mathrm{~cm}^{-2}$ & $\begin{array}{c}10 \\
(10 \text { runs of } 1 \mathrm{~h})\end{array}$ & {$\left[\mathrm{H}_{2} \mathrm{O}_{2}\right]: 8.9 \mathrm{mg} \mathrm{L}^{-1}$} & $\begin{array}{l}5.6 \mathrm{mg} \mathrm{L}^{-1} \\
(37 \% \text { loss })\end{array}$ & 24 \\
\hline $\begin{array}{c}\mathrm{CoSe}_{2} \text { on } \\
\text { carbon paper }\end{array}$ & $\begin{array}{c}0.05 \mathrm{M} \mathrm{H}_{2} \mathrm{SO}_{4} \\
(\mathrm{pH} 1.2)\end{array}$ & $0.5 \mathrm{~V}$ vs. RHE & 6 & $\begin{array}{c}\text { Faradaic efficiency: } \\
72.2 \%\end{array}$ & $53.5 \%$ & 25 \\
\hline $\begin{array}{l}\text { Anthraquinone- } \\
\text { modified } \\
\text { polyaniline }\end{array}$ & $\begin{array}{c}0.1 \mathrm{M} \\
\text { phosphate } \\
\text { buffer (pH 8.8) }\end{array}$ & $\begin{array}{c}10 \mathrm{~mA}(2.5 \\
\left.\mathrm{mA} \mathrm{cm}{ }^{-2}\right)\end{array}$ & 50 & $181 \mathrm{mmol} \mathrm{g}_{\text {catalyst }}{ }^{-1} \mathrm{hr}^{-1}$ & $181 \mathrm{mmol} \mathrm{g}_{\text {catalyst }}{ }^{-1} \mathrm{hr}^{-1}$ & $\begin{array}{l}\text { This } \\
\text { work }\end{array}$ \\
\hline
\end{tabular}

\section{References}

1. Sarapuu, A.; Vaik, K.; Schiffrin, D. J.; Tammeveski, K., Electrochemical reduction of oxygen on anthraquinone-modified glassy carbon electrodes in alkaline solution. Journal of Electroanalytical Chemistry 2003, 541, 23-29.

2. Hanna, C. M.; Luu, A.; Yang, J. Y., Proton-Coupled Electron Transfer at Anthraquinone Modified Indium Tin Oxide Electrodes. ACS Applied Energy Materials 2019, 2, (1), 59-65.

3. Zhang, G. Q.; Zhou, Y. F.; Chen, J.; Yang, F. L., Potentiostatic-Potentiodynamic Reduction Synthesis of Anthraquinone Functionalized Graphene for Oxygen Reduction in Alkaline Medium. International Journal of Electrochemical Science 2012, 7, (11), 11323-11337.

4. Jeon, T. H.; Kim, H.; Kim, H.-i.; Choi, W., Highly durable photoelectrochemical $\mathrm{H}_{2} \mathrm{O}_{2}$ production via dual photoanode and cathode processes under solar simulating and external bias-free conditions. Energy \& Environmental Science 2020, 13, (6), 1730-1742.

5. Abhayawardhana, A. D.; Sutherland, T. C., Heterogeneous proton-coupled electron transfer of a hydroxy-anthraquinone self-assembled monolayer. Journal of Electroanalytical Chemistry 2011, 653, (1), 50-55.

6. Valim, R. B.; Reis, R. M.; Castro, P. S.; Lima, A. S.; Rocha, R. S.; Bertotti, M.; Lanza, M. R. $\mathrm{V}$., Electrogeneration of hydrogen peroxide in gas diffusion electrodes modified with tertbutyl-anthraquinone on carbon black support. Carbon 2013, 61, 236-244.

7. Zhu, Q.; Pan, Z.; Hu, S.; Kim, J.-H., Cathodic Hydrogen Peroxide Electrosynthesis Using Anthraquinone Modified Carbon Nitride on Gas Diffusion Electrode. ACS Applied Energy Materials 2019, 2, (11), 7972-7979.

8. Katsoyiannis, I. A.; Canonica, S.; von Gunten, U., Efficiency and energy requirements for the transformation of organic micropollutants by ozone, $\mathrm{O}_{3} / \mathrm{H}_{2} \mathrm{O}_{2}$ and $\mathrm{UV} / \mathrm{H}_{2} \mathrm{O}_{2}$. Water Research 2011, 45, (13), 3811-3822. 
9. Hansen, K. M. S.; Andersen, H. R., Energy Effectiveness of Direct UV and $\mathrm{UV} / \mathrm{H}_{2} \mathrm{O}_{2}$ Treatment of Estrogenic Chemicals in Biologically Treated Sewage. International Journal of Photoenergy 2012, 2012, 270320.

10. Guidance Document for Integrating UV-based Advanced Oxidation Processes (AOPs) Into Municipal Wastewater Treatment Plants; Ministry of the Environment and Climate Change Showcasing Water Innovation Program (SWI): 2015.

11. Guo, K.; Wu, Z.; Yan, S.; Yao, B.; Song, W.; Hua, Z.; Zhang, X.; Kong, X.; Li, X.; Fang, J., Comparison of the $\mathrm{UV} /$ chlorine and $\mathrm{UV} / \mathrm{H}_{2} \mathrm{O}_{2}$ processes in the degradation of PPCPs in simulated drinking water and wastewater: Kinetics, radical mechanism and energy requirements. Water Research 2018, 147, 184-194.

12. Qiu, W.; Zheng, M.; Sun, J.; Tian, Y.; Fang, M.; Zheng, Y.; Zhang, T.; Zheng, C., Photolysis of enrofloxacin, pefloxacin and sulfaquinoxaline in aqueous solution by $\mathrm{UV} / \mathrm{H}_{2} \mathrm{O}_{2}, \mathrm{UV} / \mathrm{Fe}(\mathrm{II})$, and $\mathrm{UV} / \mathrm{H}_{2} \mathrm{O}_{2} / \mathrm{Fe}(\mathrm{II})$ and the toxicity of the final reaction solutions on zebrafish embryos. Science of The Total Environment 2019, 651, 1457-1468.

13. Yu, L.; Iranmanesh, S.; Keir, I.; Achari, G., A field pilot study on treating groundwater contaminated with sulfolane using $\mathrm{UV} / \mathrm{H}_{2} \mathrm{O}_{2}$. Water 2020, 12, (4), 1200.

14. Lekkerkerker-Teunissen, K.; Knol, A. H.; Derks, J. G.; Heringa, M. B.; Houtman, C. J.; Hofman-Caris, C. H. M.; Beerendonk, E. F.; Reus, A.; Verberk, J. Q. J. C.; van Dijk, J. C., Pilot Plant Results with Three Different Types of UV Lamps for Advanced Oxidation. Ozone: Science \& Engineering 2013, 35, (1), 38-48.

15. Sutherland, J.; Adams, C.; Kekobad, J., Treatment of MTBE by air stripping, carbon adsorption, and advanced oxidation: technical and economic comparison for five groundwaters. Water Research 2004, 38, (1), 193-205.

16. Chanworrawoot, K.; Hunsom, M., Treatment of wastewater from pulp and paper mill industry by electrochemical methods in membrane reactor. Journal of Environmental Management 2012, 113, 399-406.

17. El-Ashtoukhy, E. S. Z.; Amin, N. K.; Abdelwahab, O., Treatment of paper mill effluents in a batch-stirred electrochemical tank reactor. Chemical Engineering Journal 2009, 146, (2), 205-210.

18. Santos, I. D.; Dezotti, M.; Dutra, A. J. B., Electrochemical treatment of effluents from petroleum industry using a $\mathrm{Ti} / \mathrm{RuO}_{2}$ anode. Chemical Engineering Journal 2013, 226, 293299.

19. An, J.; Li, N.; Wu, Y.; Wang, S.; Liao, C.; Zhao, Q.; Zhou, L.; Li, T.; Wang, X.; Feng, Y., Revealing Decay Mechanisms of $\mathrm{H}_{2} \mathrm{O}_{2}$-Based Electrochemical Advanced Oxidation Processes after Long-Term Operation for Phenol Degradation. Environmental Science \& Technology 2020, 54, (17), 10916-10925.

20. Barazesh, J. M.; Hennebel, T.; Jasper, J. T.; Sedlak, D. L., Modular Advanced Oxidation Process Enabled by Cathodic Hydrogen Peroxide Production. Environmental Science \& Technology 2015, 49, (12), 7391-7399. 
21. Ye, Z.; Guelfi, D. R. V.; Álvarez, G.; Alcaide, F.; Brillas, E.; Sirés, I., Enhanced electrocatalytic production of $\mathrm{H}_{2} \mathrm{O}_{2}$ at Co-based air-diffusion cathodes for the photoelectroFenton treatment of bronopol. Applied Catalysis B: Environmental 2019, 247, 191-199.

22. Paz, E. C.; Aveiro, L. R.; Pinheiro, V. S.; Souza, F. M.; Lima, V. B.; Silva, F. L.; Hammer, P.; Lanza, M. R. V.; Santos, M. C., Evaluation of $\mathrm{H}_{2} \mathrm{O}_{2}$ electrogeneration and decolorization of Orange II azo dye using tungsten oxide nanoparticle-modified carbon. Applied Catalysis B: Environmental 2018, 232, 436-445.

23. Wang, Y.; Zhou, W.; Gao, J.; Ding, Y.; Kou, K., Oxidative modification of graphite felts for efficient $\mathrm{H}_{2} \mathrm{O}_{2}$ electrogeneration: Enhancement mechanism and long-term stability. Journal of Electroanalytical Chemistry 2019, 833, 258-268.

24. Zhou, W.; Rajic, L.; Chen, L.; Kou, K.; Ding, Y.; Meng, X.; Wang, Y.; Mulaw, B.; Gao, J.; Qin, Y.; Alshawabkeh, A. N., Activated carbon as effective cathode material in iron-free Electro-Fenton process: Integrated $\mathrm{H}_{2} \mathrm{O}_{2}$ electrogeneration, activation, and pollutants adsorption. Electrochimica Acta 2019, 296, 317-326.

25. Sheng, H.; Janes, A. N.; Ross, R. D.; Kaiman, D.; Huang, J.; Song, B.; Schmidt, J. R.; Jin, S., Stable and selective electrosynthesis of hydrogen peroxide and the electro-Fenton process on $\mathrm{CoSe}_{2}$ polymorph catalysts. Energy \& Environmental Science 2020, 13, (11), 4189-4203. 